\title{
The effects of cholinergic neuromodulation on neuronal phase-response curves of modeled cortical neurons
}

\author{
Klaus M. Stiefel • Boris S. Gutkin • \\ Terrence J. Sejnowski
}

Received: 3 September 2007 /Revised: 23 June 2008 / Accepted: 9 July 2008 / Published online: 11 September 2008

(C) The Author(s) 2008. This article is published with open access at Springerlink.com

\begin{abstract}
The response of an oscillator to perturbations is described by its phase-response curve (PRC), which is related to the type of bifurcation leading from rest to tonic spiking. In a recent experimental study, we have shown that the type of PRC in cortical pyramidal neurons can be switched by cholinergic neuromodulation from type II (biphasic) to type I (monophasic). We explored how intrinsic mechanisms affected by acetylcholine influence the PRC using three different types of neuronal models: a theta neuron, single-compartment neurons and a multicompartment neuron. In all of these models a decrease in the amount of a spike-frequency adaptation current was a
\end{abstract}

\footnotetext{
Action Editor: G. Bard Ermentrout

K. M. Stiefel · T. J. Sejnowski

Howard Hughes Medical Institute,

The Salk Institute for Biological Studies,

La Jolla, CA 92037, USA

B. S. Gutkin

Neural Theory Group, DEC, ENS,

CNRS and the College de France,

Paris, France

e-mail: Boris.Gutkin@ens.fr

T. J. Sejnowski

Division of Biological Sciences,

University of California at San Diego,

La Jolla, CA 92093, USA

e-mail: terry@salk.edu

Present address:

K. M. Stiefel $(\triangle)$

Okinawa Institute of Science and Technology,

Okinawa, Japan

e-mail: stiefel@salk.edu
}

necessary and sufficient condition for the shape of the PRC to change from biphasic (type II) to purely positive (type I).

Keywords Phase response curves · Cortex . Neuromodulation · Muscarine $\cdot$ Acetylcholine . Pyramidal neuron . Conductance-based model . Multi-compartmental model $\cdot$ M-current

\section{Introduction}

Acetylcholine, a central nervous system neuromodulator, is furnished to almost the entire cortex through widely diverging inputs from a number of subcortical cholinergic nuclei. At the behavioral level acetylcholine plays an important role in several aspects of cognitive function such as arousal levels, attention and vigilance, as well as memory and learning (Hasselmo 2006; Steriade 2004). One theory of learning and memory suggests that levels of cholinergic neuromodulation may switch cortical networks between learning and memory-recall modes (Hasselmo 1999). At the circuit level, cholinergic modulation appears to control the frequency content and the coherence of cortical oscillations. For example gamma-band synchronized oscillations are observed under increased cholinergic levels in the cortex (Rodriguez et al. 2004)

In vitro, experiments have shown that cholinergic antagonists promote synchronized high frequency oscillatory activity (Buhl et al. 1998; Fisahn et al. 1998). This suggests that acetylcholine has a significant modulatory control over the emergence of rhythmic activity in cortical circuits. Certainly such effects are in part due to the acetylcholine modulation of synaptic transmission (Auerbach and Segal 1996) and plasticity (Artola and Singer 1993; 
Stiefel et al. 2005) and in part due to cholinergic modulation of intrinsic cell excitability (McCormick 1993). Cholinergic modulation affects a variety of both ionotropic (nicotinic) and metabotropic (muscarinic) receptor targets (Schoebesberger et al. 2000). Acetylcholine acting on muscarinic receptors downregulates several slow potassium currents ( $I_{\mathrm{KM}}, I_{\mathrm{KAHP}}$ (Madison et al. 1987; McCormick 1993)), and changes the input resistance by down regulating the leak conductance. These currents control cellular excitability and repetitive firing of cortical pyramidal neurons.

Given such diverse acetylcholine action at the single cell level, an interesting issue is which of the effects mediated by the muscarinic acetylcholine-receptor modulation can be responsible for the emergence of collective behaviors, such as synchrony and oscillations. In fact, previous theoretical work has hinted that suppression of slow cation channels can bias pyramidal neurons toward synchrony with excitatory synaptic coupling by modulating the dynamics of spike generation (Crook et al. 1998a, b; Ermentrout et al. 2001). In this report our goal is to systematically examine the various mechanisms of cholinergic action and to identify those that have the greatest impact on the dynamics of spike generation in cortical neurons.

We focus on the cholinergic effects on the excitability of regular spiking (pyramidal) neurons that can be described as oscillators. Specifically, we examine the dynamics underlying the spike generation and how the cell goes from excitable to periodically firing. Knowing the type of this transition can provide insight not only about the inputoutput function of the neurons (the FI curve) but also about the potential presence of subthreshold oscillations as well as the synaptic mechanisms that can potentially lead to synchrony (Ermentrout 1996). This transition from rest (a stable fixed point and a threshold) to spiking (following a limit cycle) can occur via two major dynamical mechanisms: the "saddle-node on an invariant cycle" bifurcation (from here on referred to as "saddle-node" bifurcation) and the subcritical Hopf-Andronov bifurcation. In the case of the saddle-node bifurcation, a stable fixed point (a node) and a saddle-point collide into a saddle-node and disappear to leave a stable limit cycle. In the case of the subcritical Hopf-Andronov bifurcation, the loss of stability occurs when the real parts of conjugate eigenvalues grow positive, hence destabilizing the fixed point (see Izhikevich (2007) for further discussion). These different bifurcations affect the shape of the FI curve, which is continuous for the SNIC bifurcation and discontinuous for the Hopf-Andronov case. Furthermore Hopf-Andronov systems can exhibit significant subthreshold oscillations, and hysteresis.

These two classes of bifurcations also result in qualitatively different phase response curves (PRCs). The PRCs characterize the phase shift the results when a neuronal oscillator is perturbed by an input as a function of the phase of the perturbation. (Although the PRC can be constructed for both excitatory and inhibitory perturbations, the standard convention is to report results for depolarizing inputs.) In the case of a saddle-node bifurcation, the PRC is purely positive, or type I (except possibly for an arbitrarily small region near the origin Hansel and Mato 1995), with a phase advance in response to perturbations at all phases (Ermentrout 1996). In the case of a Hopf-Andronov bifurcation, the PRC is biphasic, or type II, with a phase delay in response to perturbations early in the period and a phase advance in response to perturbations late in the period (Hansel and Mato 1995; Ermentrout 1996; Moehlis et al. 2006).

Computational studies have previously observed that under block of slow voltage dependent potassium currents $\left(I_{\mathrm{M}}\right)$, the PRC changed quantitatively, associated with a change in the bifurcation structure (Ermentrout et al. 2001). The PRCs went from biphasic with full current conductance to a monophasic when the current was sufficiently weakened. Gutkin et al. (2005) further hypothesized that the differences seen in the PRCs of various pyramidal neurons experimentally (Reyes and Fetz 1993) can be explained by the relative levels of the slow low-threshold voltage-dependent potassium current. Modeling studies also suggest that block of the high threshold, $\mathrm{Ca}^{2+}$ dependent slow potassium current $\left(I_{\mathrm{AHP}}\right)$ cannot change the PRC type, but affects only the relative skew of the PRC peak. We thus hypothesized that the $I_{\mathrm{M}}$ is the key component necessary to induce the switch of PRC type. In a recent experimental study, cholinergic neuromodulation shifted the type of PRC from type II to type I in cortical pyramidal neurons (Stiefel et al. 2003). The decrease in the $I_{\mathrm{M}}$ current leads to a decrease in spike-frequency adaptation and is also a prime candidate for the switch from type II to type I PRC.

However, as pointed out above, acetylcholine changes a multitude of mechanisms, so modulation of $I_{\mathrm{M}}$ may not be a necessary or sufficient factor in switching the PRC type. We address this question systematically with simulations using successively more complex neuronal models. The first model is the theta-neuron, for which the switch of PRCs can be shown directly. We then examine a simple model (containing a restricted set of voltage dependent channels) and a more complex single-compartment model (with a larger set of channels) and a multi-compartment neuron.

For each of the above model neurons we show that the sum total of cholinergic effects causes a switch of the PRC. We further identified the down-regulation of a lowthreshold spike-frequency adaptation current $\left(I_{\mathrm{M}}\right)$ as the cause for the PRC type to switch from type II to type I, and that the other mechanisms are not able to induce the switch. 


\section{Methods}

All simulations were carried out with the NEURON computer program (Hines and Carnevale 1997, 2000). The simulations used the CNexp numerical integration method with $\mathrm{dt}=0.025 \mathrm{~ms}$. The source code for these simulations (NEURON hoc and mod files) containing all simulation parameters is available as supplementary material or by request from K.M.S. and can be obtained from the SenseLab model database (http://senselab.med.yale.edu/modeldb/). All simulations were run in NEURON version 5.x either under Windows XP on an Athlon AMD 64 processor or under FreeBSD 4.4 on a Pentium IV processor.

We used four different neuronal models, with increasing dimensionality. These were the theta neuron $(D=2)$, a simple single compartment neuron $(D=5)$, a more complex singlecompartment neuron $(D=9)$ and a multi-compartment neuron $(D>100)$. The latter neuron model contained 1,393 compartments with up to six currents per compartment (see below). Because the currents in the different compartments are not independent (and thus not every state variable is a separate dimension), the dimensionality is ill defined.

\subsection{Theta-neuron}

The theta-neuron, the simplest neural model that spikes, is an oscillator with a single phase variable, $\theta$ (see e.g. Gutkin and Ermentrout 1998). The addition of an adapting current makes it a two-dimensional model following the equations:

$\frac{d \Theta}{d t}=1-\cos \Theta+(1+\cos \Theta)\left(\beta+s \bar{g}_{\mathrm{M}}+S\right)$

$\tau_{\mathrm{M}} \frac{d s}{d t}=\left(k_{\mathrm{M}} \Theta\right)(1-s)-s$

$k_{\mathrm{M}}=a e^{-b(1-\cos (\Theta-\Theta \mathrm{th}))}$

where in Eq. (1) is $\beta$ the tonic drive, $S$ the synaptic drive; in Eq. (2) $\mathrm{s}$ is the state variable for the adaptation, $\Theta_{\mathrm{th}}, b, g_{\mathrm{M}}$ and $\tau_{\mathrm{M}}$ are threshold, activation slope, maximal conductance and time-constant of the adapting current respectively.

\subsection{Simple single-compartment neuron}

The single-compartmental neuron model was geometrically simple, but included biophysically realistic mechanisms for neuronal spiking based on Hodgkin and Huxley (1952). This model pyramidal neuron had identical equations for the current kinetics as in (Golomb and Amitai 1997) but included, only the fast $\mathrm{Na}^{+}$, leak, delayed rectifier $\mathrm{K}^{+}$ currents, and a slower $\mathrm{K}^{+}$current $\left(I_{\text {Kslow }}\right)$, responsible for spike frequency adaptation. This last current was an amalgam of low threshold slow potassium currents, of which $I_{\mathrm{M}}$ is an example. Since we did not adjust the parameters to fit precisely the data on the muscarinic sensitive $I_{\mathrm{M}}$, we refer to this current generically as $I_{\text {Kslow }}$. The voltage equation is:

$$
\begin{aligned}
C_{\mathrm{M}} \frac{d V}{d t}= & -m^{3} h \bar{g}_{\mathrm{Na}}\left(V-E_{\mathrm{Na}}\right)-n \bar{g}_{\mathrm{KDR}}\left(V-E_{\mathrm{K}}\right) \\
& -s \bar{g}_{\mathrm{Ks}}\left(V-E_{\mathrm{K}}\right)-\bar{g}_{\text {leak }}\left(V-E_{\text {leak }}\right)-I_{\text {inj }},
\end{aligned}
$$

with $m, n, h$ and $s$ the voltage-dependent activation variables that follow equations of the form:

$\frac{d x}{d t}=\tau(V)\left(x-x_{\infty}(V)\right)$,

where $\tau(V)$ and $x_{\infty}(V)$ are experimentally determined functions for the time constant and infinite activation state of the state variables, $C_{\mathrm{m}}$ the membrane capacitance, $V$ the membrane potential, $m, h, n$ and $s$ the state variables of the $\mathrm{Na}^{+}$current activation/inactivation and the delayed rectifier/ slow $\mathrm{K}^{+}$current, $g_{\mathrm{Na}}, g_{\mathrm{KDR}}, g_{\mathrm{Ks}}$ and $g_{\text {leak }}$ the conductances of the respective currents, and $E_{\mathrm{Na}}, E_{\mathrm{K}}$ and $E_{\text {leak }}$ their respective reversal potentials. $I_{\mathrm{inj}}$ is the injected current.

\subsection{Complex single-compartment neuron}

The single-compartment model of neuronal spiking is an expanded version of a previous model (Golomb and Amitai 1997, Eqs. (4-5)). $I_{\mathrm{M}}$ and $I_{\mathrm{AHP}} \mathrm{K}^{+}$currents were included to generate an after-hyperpolarization (AHP). Because the $I_{\mathrm{AHP}}$ is $\mathrm{Ca}^{2+}$-dependent, an $I_{\mathrm{L}} \mathrm{Ca}^{2+}$-current and an exponential $\mathrm{Ca}^{2+}$ decay representing a $\mathrm{Ca}^{2+}$-buffer were also included. Also, a hyperpolarization-activated mixed cation (depolarizing) current, $I_{\mathrm{H}}$, and an inactivating fast $\mathrm{K}^{+}$-current, $I_{\mathrm{KA}}$, were included. All these currents occur in cortical pyramidal neurons (Johnston and $\mathrm{Wu}$ 1994). The formalisms for the currents are identical to the previous model. Note that this model simulates neuronal dynamics of both the membrane potential and the $\mathrm{Ca}^{2+}$ concentration. The formalism for the $\mathrm{Ca}^{2+}$ dynamics follows:

$\frac{d \mathrm{Ca}^{2+}}{d t}=\frac{2 I_{\mathrm{Ca}^{2+}}}{\delta}+\frac{\left(\mathrm{Ca}_{0}^{2+}-\mathrm{Ca}^{2+}\right)}{\tau_{\mathrm{Ca}^{2+}}}$,

with $F$ the Faraday constant $(96,500 \mathrm{C})$ and $\delta$ the fraction of the intracellular space affecting the $\mathrm{Ca}^{2+}$ dependent current (a $1 \mu \mathrm{m}$ shell). This term couples the $\mathrm{Ca}^{2+}$ current to the $\mathrm{Ca}^{2+}$ concentration, which in the absence of any influx relaxes back to $\mathrm{Ca}^{2+}{ }_{0}$ with the time constant $\tau_{\mathrm{Ca} 2+}=35 \mathrm{~ms}$.

\subsection{Complex multi-compartment neuron}

This model, in contrast to the previous ones, incorporates the spatial structure of the dendrites and is based on a 
reconstructed cortical layer II pyramidal neuron (Mainen and Sejnowski 1995). The shape of the neuron is depicted in Fig. 4(a). The neuronal morphology had 257 compartments. When investigating the effects of local dendritic changes, the number of compartments was increased to 1,393 , to assure that each compartment was sufficiently small $(<8 \mu \mathrm{m})$ to be approximately isopotential.

The voltage equation for each compartment contained an additional term for current flow from the neighboring compartments (for the dendritic compartment $n$ ):

$C_{\mathrm{m}} \frac{d V_{n}}{d t}=\sum I-I_{\mathrm{inj}}-g_{\mathrm{ax}}\left(V_{n}-V_{n-1}\right)-g_{\mathrm{ax}}\left(V_{n+1}-V_{n}\right)$,

where $g_{\text {ax }}$ is the axial conductance and $\Sigma I$ is the sum of all intrinsic ionic currents. The model consisted of axonal, somatic and dendritic regions with distinct properties. These membrane properties were adjusted from their original values in order to more realistically reproduce the spike and AHP-shape and pattern seen in our recordings. Specifically, we increased the somato-dendritic conductances $g_{\mathrm{M}}=2 \times{ }^{-4} \mathrm{~S} \mathrm{~cm}^{-2}$ and $g_{\text {leak }}=2 \times 10^{-5} \mathrm{~S} \mathrm{~cm}^{-2}$ and decreased the somatic and somato-dendritic conductances to $g_{\mathrm{AHP}(\mathrm{Ca})}=1 \times 10^{-2} \mathrm{~S} \mathrm{~cm}^{-2}$ and $g_{\mathrm{CaL}}=1 \times 10^{-5} \mathrm{~S} \mathrm{~cm}^{-2}$. In addition, the time constant of the $\mathrm{Ca}^{2+}$-decay was decreased to $35 \mathrm{~ms}$ to reproduce the shape of the recorded AHP more closely and to match the complex singlecompartment model. $g_{\mathrm{KDR}}$ in the soma was decreased from 200 to $100 \mathrm{~S} \mathrm{~cm}^{-2}$. The axonal conductances remained unchanged.

\subsection{Determination of phase-response curves}

We determined the PRCs in all models by first simulating spiking until the spike discharge frequency reached stationarity $\left(\mathrm{ISI}_{n} / \mathrm{ISI}_{n-1}<1.01\right)$. Then we perturbed the voltage by injecting a small $(<1 \mathrm{mV})$ and short $(<20 \mathrm{~ms})$ exponentially decaying depolarizing synaptic event. The $\Delta t$ of the ISI as a result of the synaptic input was determined and normalized to the control (unperturbed) ISI duration. This was repeated at 64 phase positions spanning the entire period of the oscillation. Changing conductances to mimic cholinergic effects can alter (increase) the firing frequency of the neuron. When we intended to keep the firing frequency of the model neuron constant during a parameter sweep, we determined a firing frequency/current curve (FI curve) for each parameter value and used it as a lookuptable to determine the appropriate current for the desired frequency.

\section{Results}

\subsection{Theta-neuron}

Using the theta-neuron, we modeled the effects of cholinergic modulation by decreasing the adaptation current $\left(g_{\mathrm{m}}\right.$ from 1 to 0.7 ) and by increasing the drive ( $\beta$ from 0.5 to 0.52 ). These manipulations mimicked the experimentally observed decrease in $I_{\mathrm{M}}$, the depolarization and the increase in the input resistance. In response to these manipulations, the steady-state firing frequency increased from 8.6 to $69.1 \mathrm{~Hz}$ and the PRC of the theta neuron switched from type II to type I (Fig. 1(b, c)). This switch from type II to type I was observed both when lowering $g_{\mathrm{m}}$ (1 to 0.6 , Fig. 1(d), top) and when increasing $\beta$ (0.49 to 1, Fig. 1(d), bottom). During these parameter sweeps, the firing frequency increased from 10.2 to $93 \mathrm{~Hz}$ and from 5.8 to $196 \mathrm{~Hz}$, respectively.

High firing frequencies can mask type II dynamics when the frequency is so high that the dynamics of the adaptation current become much slower than the spiking dynamics. We thus performed a parameter sweep over $g_{\mathrm{m}}$ ( 1 to 0.6$)$ with $\beta$ adjusted to keep the firing frequency at $10 \pm 2 \mathrm{~Hz}$ (Fig. 1(d), center). With decreasing $g_{\mathrm{m}}$, the negative part of the PRC decreased and its maximum, minimum and zerocrossing shifted to the left (earlier parts of the phase). The zero-crossing, when present, also shifted to the left. This shows that a change in $g_{\mathrm{m}}$ is sufficient to alter the PRC of the theta neuron.

\subsection{Simple single-compartment neuron}

To model cholinergic neuromodulation in a single compartment model with $I_{\text {leak }}, I_{\mathrm{Na}}, I_{\mathrm{KDR}}$ and $I_{\mathrm{Kslow}}$, we altered the leak conductance $\left(g_{\text {leak }}, 2 \times 10^{-5}\right.$ to $\left.1.8 \times 10^{-5} \mathrm{~S} \mathrm{~cm}^{-2}\right)$, reversal potential of the leak current ( $E_{\text {leak }},-60$ to $-58 \mathrm{mV}$ ) and the conductance of $I_{\text {Kslow }}\left(g_{\text {Kslow }}, 2 \times 10^{-3}\right.$ to $\left.0 \mathrm{~S} \mathrm{~cm}^{-2}\right)$. These modifications led to a switch of the PRC of the model neuron from type II to type I (Fig. 2(b)).

We conducted parameter sweeps over all the parameters manipulated to model cholinergic neuromodulation, both with all other parameters kept at default values and with the injected current adjusted to keep the firing frequency at $10 \pm 1 \mathrm{~Hz}$ ( $g_{\text {leak }}: 1.6 \times 10^{-5}$ to $2.2 \times 10^{-5} \mathrm{~S} \mathrm{~cm}^{-2}, E_{\text {leak }}:-64$ to $56 \mathrm{mV}, g_{\text {Kslow }}: 0$ to $2.2 \times 10^{-3} \mathrm{~S} \mathrm{~cm}^{-2}$ ). We found that only a change in the value of $g_{\mathrm{m}}$ lead to a qualitative change of the PRC, a switch from type II to I. This was observed both with and without an adjustment of the injected current to keep the firing frequency at $10 \mathrm{~Hz}$. In both cases, the maximum, minimum and zero-crossing of the PRC shifted

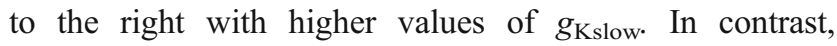


(a)

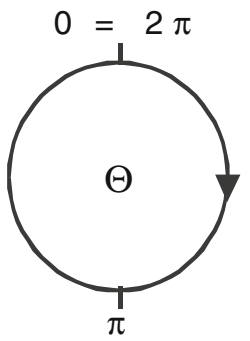

(b)
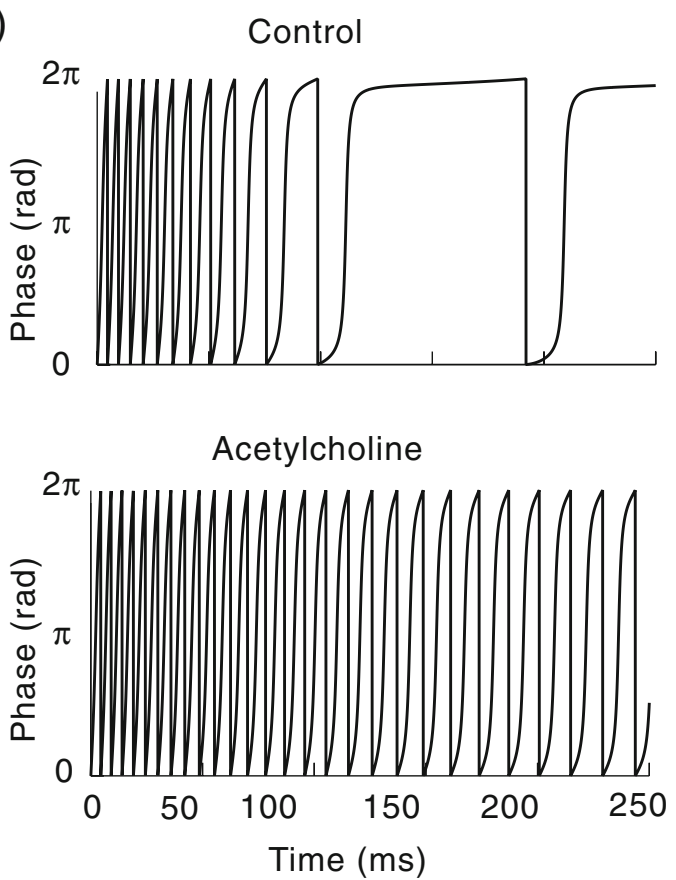

(c)

\section{Control/ Acetylcholine}

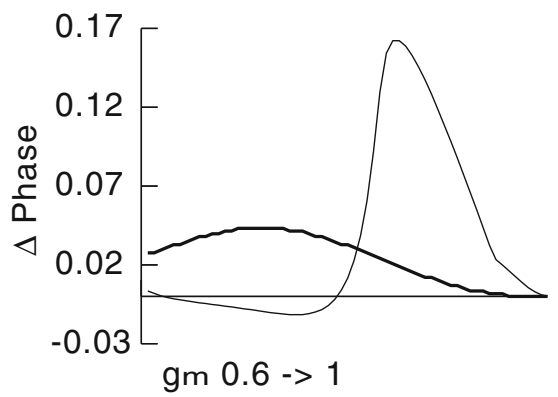

Fig. 1 Theta-neuron. (a) Schematic drawing of the model. (b) Voltage traces with standard parameters $\left(g_{\mathrm{m}}=1, \beta=0.5\right)$ and with parameter modifications simulating cholinergic neuromodulation $\left(g_{\mathrm{m}}=0.7, \beta=\right.$ 0.52). (c) Phase response curves (PRCs) with standard (thin line) and (d)
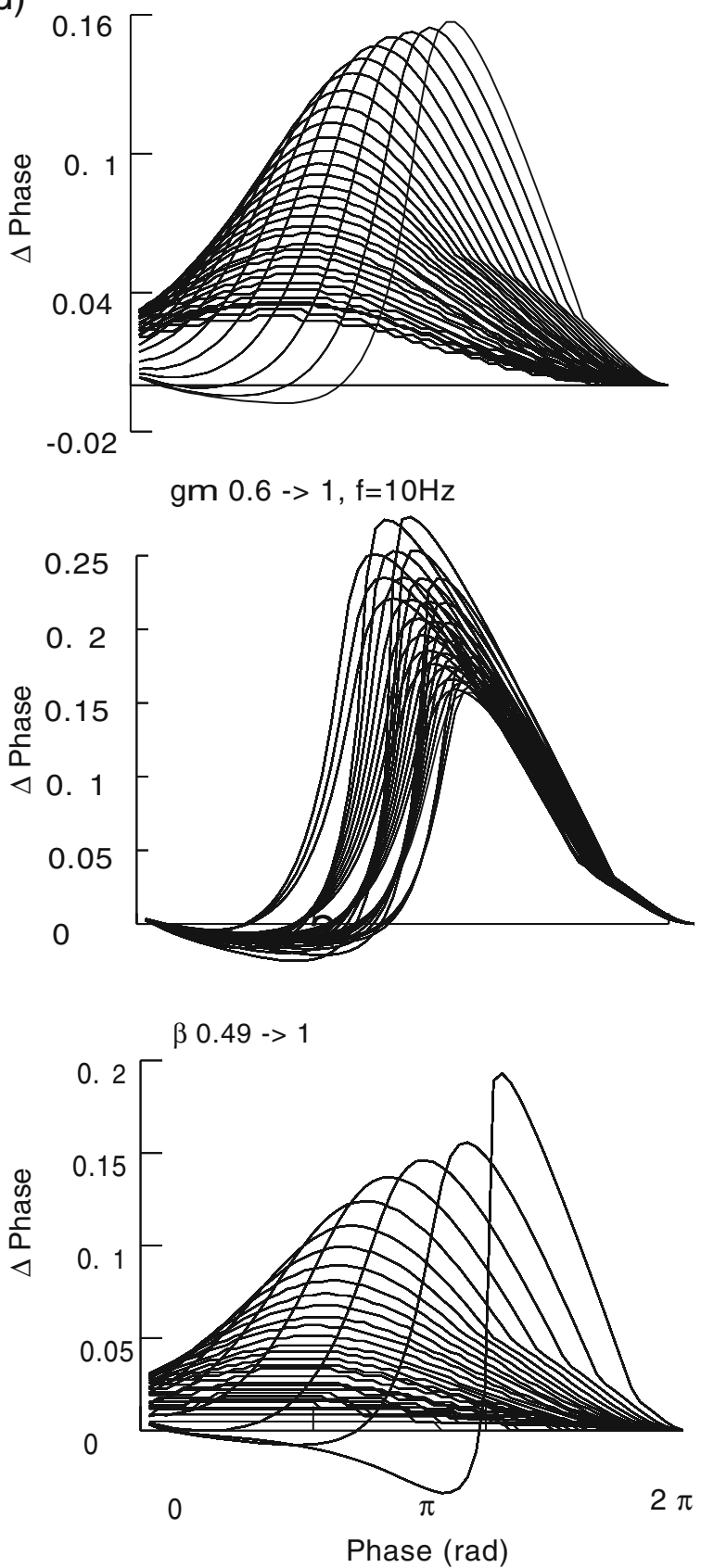

cholinergic (thick line) parameters. (d) (top): $g_{\mathrm{m}}$ varied from 0.6 to 1.0; (middle): $g_{\mathrm{m}}$ varied from 0.6 to 1.0 with $\beta$ adjusted to keep the firing rate at $10 \pm 2 \mathrm{~Hz}$.(bottom): $\beta$ varied from 0.49 to 1.0

and maxima. This effect disappeared when the firing frequency was kept constant by adjusting the injected current. Changing $E_{\text {leak }}$ and $g_{\text {leak }}$ had little influence on the PRC. 
(a)

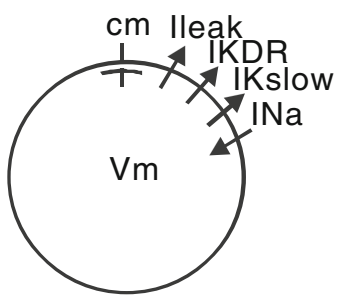

(b)

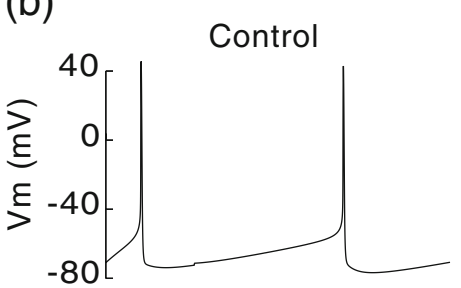

Acetylcholine

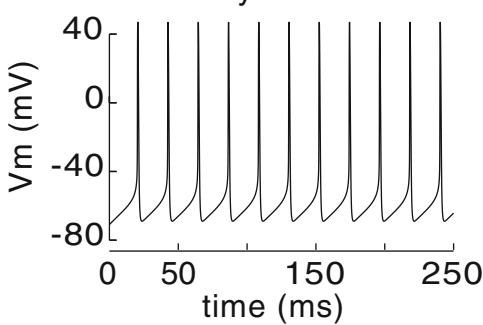

(c) Control / Acetylcholine

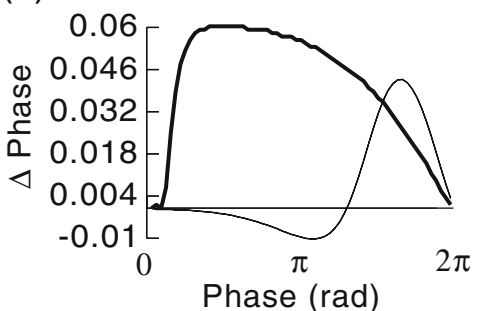

Fig. 2 Simple single-compartment neuron. (a) schematic drawing of the model. (b) Voltage traces with standard parameters $\left(E_{\text {leak }}=\right.$ $-60 \mathrm{mV}, g_{\text {leak }}=2 \times 10^{-5} \mathrm{~S} \mathrm{~cm}^{-2}, g_{\text {Kslow }}=0.002 \mathrm{~S} \mathrm{~cm}^{-2}$ ) and with parameter modifications simulating cholinergic effects $\left(E_{\text {leak }}=\right.$ $-58 \mathrm{mV}, g_{\text {leak }}=1.8 \times 10^{-5} \mathrm{~S} \mathrm{~cm}^{-2}, g_{\text {Kslow }}=0 \mathrm{~S} \mathrm{~cm}^{-2}$ ). (c) PRCs with standard (thin line) and cholinergic (thick line) parameters. (d) PRCs
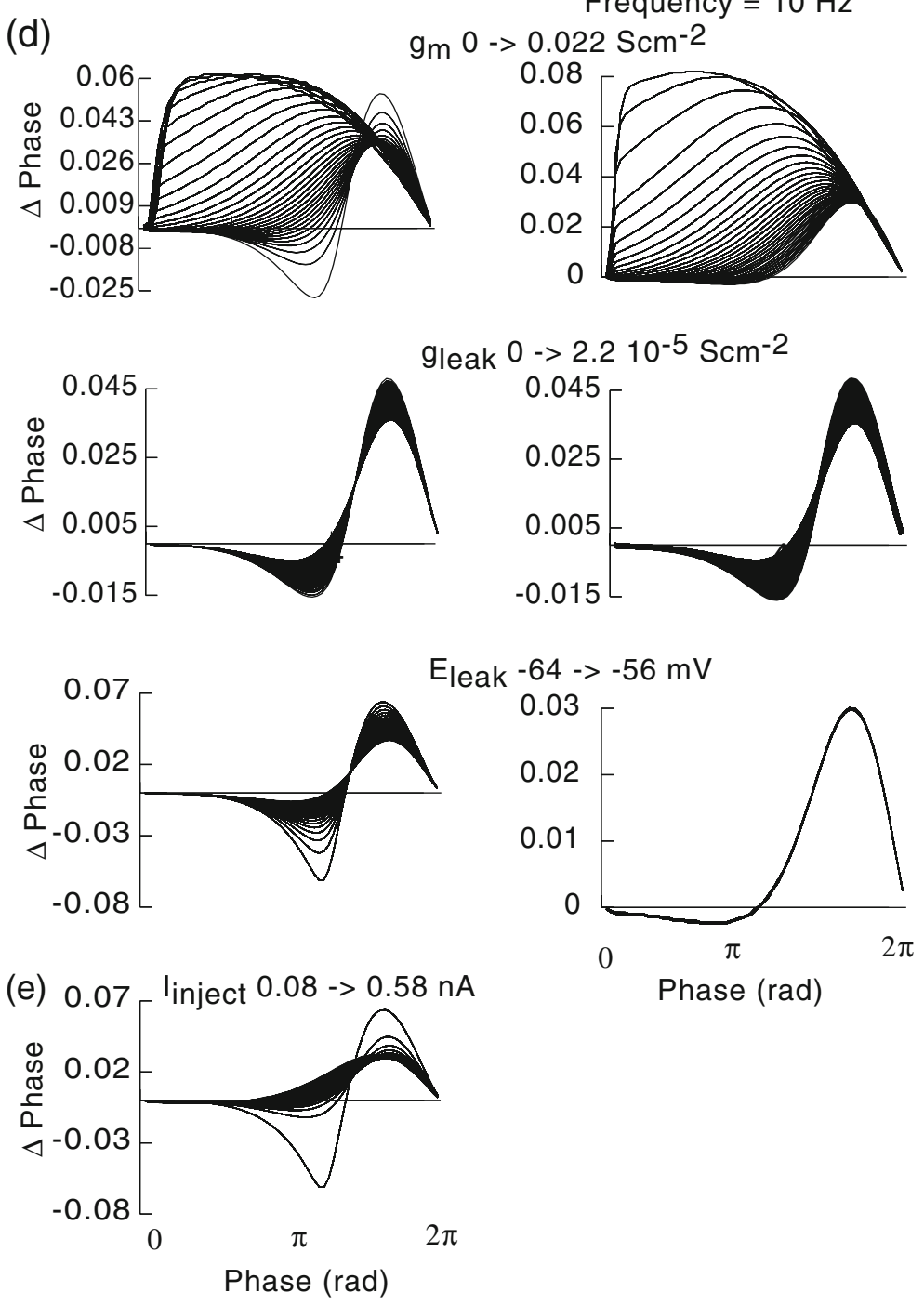

Eleak $-64->-56 \mathrm{mV}$

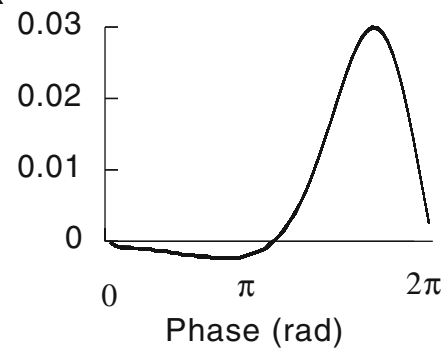

with $g_{\text {Kslow }}$ varied from 0 to $0.0022 \mathrm{~S} \mathrm{~cm}^{-2}$ (top), $g_{\text {leak }}$ varied from $1.6 \times 10^{-5}$ to $2.2 \times 10^{-5} \mathrm{~S} \mathrm{~cm}^{-2}$ (middle) and $E_{\text {leak }}$ varied from -64 to $-58 \mathrm{mV}$ (bottom). Left column: all other parameters kept constant. Right column: $I_{\text {inject }}$ adjusted to keep the firing rate at $10 \pm 1 \mathrm{~Hz}$. (e) PRCs with $I_{\text {inject }}$ varied from 0.08 to $0.58 \mathrm{nA}$

The effect of cholinergic neuromodulation was modeled by altering the conductance ( $g_{\text {leak }}$, from $2 \times 10^{-5}$ to $1.8 \times$ $10^{-5} \mathrm{~S} \mathrm{~cm}^{-2}$ ) and reversal potential ( $E_{\text {leak }}$, from -60 to $-58 \mathrm{mV}$ ) of the leak current and the conductance of $I_{\mathrm{KM}}$ and $I_{\text {KAHP. }}$ Madison et al. (1987) showed that in hippocampal pyramidal neurons, these two currents are both cholinergically modulated, albeit with different sensitivities. $I_{\mathrm{KAHP}}$ is inhibited by cholinergic modulation at about ten times lower concentrations than $I_{\mathrm{KM}}\left(\mathrm{IC}_{50}=0.3\right.$ and $5 \mu \mathrm{M}$ for carbachol). Assuming that superficial neocortical neurons would react similarly, we simulated two different conditions: lowconcentration cholinergic modulation $\left(I_{\mathrm{M}}\right.$ down-regulated 

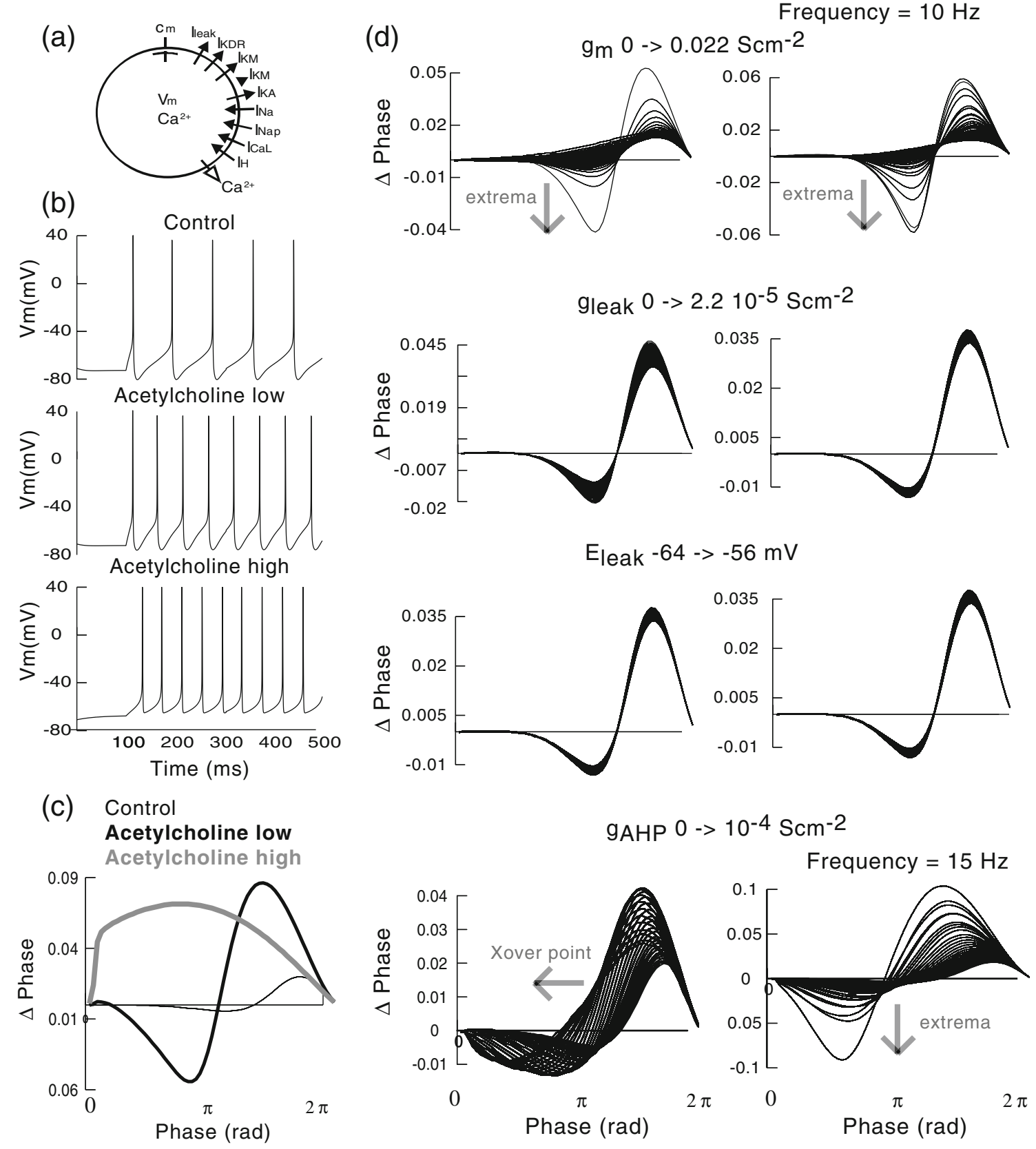

Fig. 3 Complex single-compartment neuron. (a) Schematic drawing of the model. (b) Voltage traces with standard parameters $\left(E_{\text {leak }}=-60 \mathrm{mV}\right.$, $g_{\text {leak }}=2 \times 10^{-5} \mathrm{~S} \mathrm{~cm}^{-2}, g_{\mathrm{KM}}=5 \times 10^{-4} \mathrm{~S} \mathrm{~cm}^{-2}, g_{\mathrm{AHP}}=1 \times 10^{-4} \mathrm{~S} \mathrm{~cm}^{-2}$ ), with parameter modifications simulating low-concentration cholinergic neuromodulation $\left(E_{\text {leak }}=-58 \mathrm{mV}, g_{\text {leak }}=1.8 \times 10^{-5} \mathrm{~S} \mathrm{~cm}^{-2}, g_{\mathrm{KM}}=4.5 \times\right.$ $\left.10^{-4} \mathrm{~S} \mathrm{~cm}^{-2}, g_{\mathrm{AHP}}=2.5 \times 10^{-5} \mathrm{~S} \mathrm{~cm}^{-2}\right)$ and parameters simulating lowconcentration cholinergic neuromodulation $\left(E_{\text {leak }}=-58 \mathrm{mV}, g_{\text {leak }}=1.8 \times\right.$ $10^{-5} \mathrm{~S} \mathrm{~cm}^{-2}, g_{\mathrm{KM}}=2.5 \times 10^{-5} \mathrm{~S} \mathrm{~cm}^{-2}, g_{\mathrm{AHP}}=0 \mathrm{~S} \mathrm{~cm}^{-2}$ ). (c) PRCs with standard (thin line), low-concentration cholinergic (thick black line) and

by $10 \%$ to $4.5 \times 10^{-4}$ and $I_{\mathrm{AHP}}$ down-regulated by $75 \%$ to $2.5 \times 10^{-5} \mathrm{~S} \mathrm{~cm}^{-2}$ ) and high-concentration cholinergic modulation $\left(I_{\mathrm{M}}\right.$ down-regulated by $95 \%$ to $5 \times 10^{-5}$ and $I_{\mathrm{AHP}}$ down-regulated by $100 \%$ to $0 \mathrm{~S} \mathrm{~cm}^{-2}$ ). Unlike the previous simpler model, in this model spiking was coupled to the $I_{\mathrm{AHP}}$ induced after-hyperpolarization not only directly, via the high-concentration cholinergic (thick gray line) parameters. (d) PRCs with $g_{\mathrm{KM}}$ varied from 0 to $0.0006 \mathrm{~S} \mathrm{~cm}^{-2}$ (top), $g_{\text {leak }}$ varied from $1.6 \times$ $10^{-5}$ to $2.2 \times 10^{-5} \mathrm{~S} \mathrm{~cm}^{-2}$ (middle) and $E_{\text {leak }}$ varied from -64 to $-58 \mathrm{mV}$ (bottom), $g_{\mathrm{AHP}}$ varied from 0 to $1 \times 10^{-4} \mathrm{~S} \mathrm{~cm}^{-2}$. Left column: all other parameters kept constant. Right column: PRCs with $I_{\text {inject }}$ adjusted to keep the firing rate at $10 \pm 1 \mathrm{~Hz}(15 \pm 1 \mathrm{~Hz}$ in the last row, as the minimum firing frequency with $g_{\mathrm{AHP}}=0$ is $>13 \mathrm{~Hz}$ ). The gray arrows depict the direction of the trends along the parameter sweeps

voltage, but also indirectly, via $\mathrm{Ca}^{2+}$ influx. The set of newly introduced ionic currents might change the parameter dependence of the model's PRC.

Interestingly, simulation of low concentration cholinergic modulation led to a PRC that was even more pronouncedly biphasic than under control conditions. In contrast, the 
simulation of high-concentration cholinergic modulation produced a transition from a type II to a type I PRC (Fig. 3(c); the firing frequencies in these simulations were kept at $12 \pm 1 \mathrm{~Hz}$ in order to ensure comparability).

Parameter sweeps $g_{\text {leak }}, E_{\text {leak }}, g_{\mathrm{M}}$, and $g_{\mathrm{AHP}}\left(g_{\text {leak }}\right.$ : from $1.6 \times 10^{-5}$ to $2.2 \times 10^{-5} \mathrm{~S} \mathrm{~cm}^{-2}, E_{\text {leak }}$ : from -64 to $56 \mathrm{mV}$, $g_{\mathrm{M}}$ : from 0 to $6 \times 10^{-4} \mathrm{~S} \mathrm{~cm}^{-2}, g_{\mathrm{AHP}} 0$ to $10^{-4} \mathrm{~S} \mathrm{~cm}^{-2}$ ) revealed that the qualitative change in the PRC was solely a consequence of changes in $g_{\mathrm{M}}$. This occurred regardless of whether the firing frequency was kept constant by adjusting current injection. No switch from a biphasic to a purely positive PRC occurred when $g_{\text {leak }}, I_{\text {leak }}$ or $g_{\text {AHP }}$ were varied. The sweeps over the parameters of the leak current had little effect on the PRCs. Increasing $g_{\mathrm{AHP}}$ moved the crossover point between the negative and the positive part of the PRC to the left (towards earlier phases). When the firing frequency during the determination of the PRCs was kept constant, this effect was greatly reduced and the extrema of the PRCs decreased with an increasing amount of $g_{\text {AHP. }}$

In conclusion, the effects of cholinergic neuromodulation on the PRC were biphasic in simulations of the complex single-compartmental model. An increase of the negative part of the PRC mediated by low modulator concentration was followed by a disappearance of this negative part mediated by a high acetylcholine concentration. The switch from a type II to a type I PRC was due to the decrease of $I_{\mathrm{M}}$. However, under control conditions, the $I_{\mathrm{AHP}}$ masked a part of the negative portion of the PRC.

\subsection{Complex multi-compartment neuron}

The most complex model used in this study was a multicompartment model of a cortical layer II/III pyramidal neuron (Fig. 4(a)) which matched our in vitro experiments (Stiefel et al. 2003). The model included spatial complexity (soma, dendrites and axon), several ionic conductances distributed heterogeneously over the different parts of the neuron and spike initiation in the axon (Stuart et al., 97, Fig. 4(b)). Using this model we simulated the effect of low and high concentrations of cholinergic neuromodulation by implementing the following parameter changes in the dendrites: To mimic low concentrations, we changed $E_{\text {leak }}=-60$ to $-58 \mathrm{mV}, g_{\text {leak }}=210^{-5}$ to $1.8 \times 10^{-5} \mathrm{~S} \mathrm{~cm}^{-2}, g_{\mathrm{KM}}=2 \times$ $10^{-4}$ to $1.8 \times 10^{-4} \mathrm{~S} \mathrm{~cm}^{-2}$ and $g_{\mathrm{AHP}} 3 \times 10^{-4}$ to $7.5 \times 10^{-5} \mathrm{~S}$ $\mathrm{cm}^{-2}$. To mimic high concentrations, we changed $E_{\text {leak }}=-60$ to $-58 \mathrm{mV}, g_{\text {leak }}=2 \times 10^{-5}$ to $1.8 \times 10^{-5} \mathrm{~S} \mathrm{~cm}^{-2}, g_{\mathrm{KM}}=2 \times 10^{-4}$ to $10^{-3} \mathrm{Scm}^{-2}$. Cantrell et al. (1996) reported a significant muscarinic acetylcholine-receptor mediated down-regulation of sodium current conductance. Interestingly, they found two populations of neurons, in which this current was reduced to $90 \%$ and $55 \%$, respectively. Hence, we globally (dendrites, soma and axon) decreased $g_{\mathrm{Na}}$ to $55 \%$ of its original value.
Reducing $g_{\mathrm{Na}}$ to only $90 \%$ of its initial value caused the model neurons to spike in doublets (Fig. 4(c)), a behavior also observed in response to the application of high carbachol concentrations in vitro (unpublished observations). We studied the $55 \%$ reduction to focus on the PRCs of individual spikes rather than doublets.

Initially we determined the PRC by a synapse located on an intermediate part of the apical dendritic tuft (Fig. 4(d)). The PRC under control conditions was biphasic (type II), and became even more saliently biphasic when low concentrations of cholinergic neuromodulation (reducing $I_{\mathrm{M}}$ and strongly reducing $\left.I_{\mathrm{AHP}}\right)$ were simulated. When simulating high concentrations of cholinergic neuromodulation (strongly reducing $I_{\mathrm{M}}$ and abolishing $I_{\mathrm{AHP}}$ ), the PRC became monophasic (type I). This switch was observed when the value of $g_{M}$ was changed in isolation, but not when the values of $g_{\text {leak }}, E_{\text {leak }}$, or $g_{\mathrm{AHP}}$ were changed (Fig. 4(e)). Whereas the effects of changing the parameters of the passive conductance were minimal, reducing $I_{\mathrm{AHP}}$ reduced the negative part of the PRC and shifted it to an earlier part of the phase. These observations further strengthened the importance of $I_{\mathrm{M}}$ in determining the type of PRC displayed by the neuron.

In order to explore the role of the spatial location of the synapse we determined the PRC by synapses on three different locations, ranging from the proximal primary to a tertiary dendrite (Fig. 5(a), synapse 2 is identical to the previously investigated synapse). As the spike initiation takes place in the axon, the synapses where chosen progressively farther from the initiation site. The currents from the dendritic synapses have to pass through active and passive cable filtering by the dendrites and the soma (a large sink), before reaching this spike initiation site. All these factors could potentially influence how the investigated synapses affect the PRCs (Goldberg et al. 2007). The PRCs determined for all three synapses were qualitatively similar. The PRCs under control conditions were biphasic (type II), even more so than when simulating low concentrations, and monophasic (type I) when simulating high concentrations of cholinergic neuromodulation (Fig. 5(a)).

Fig. 4 Complex multi-compartment neuron. (a) Model neuron morphology and synapse location, indicated by circles. (b) Timecourse of action potential initiation at different cellular locations. Soma: Black, first node of Ranvier: red, axon hillock: Blue, axon initial segment: Pink. (c) Voltage traces with standard parameters and with parameter modifications simulating low and high concentration cholinergic neuromodulation, with a strong and moderate reduction in $g_{\mathrm{Na}}\left(I_{\text {injected }}=0.12,0.12,0.03,0.3 \mathrm{nA}\right)$. (d): PRCs with control (thin line), low concentration cholinergic (thick line) and high concentration cholinergic parameters (gray line). Firing frequencies were kept at $7 \pm$ $1 \mathrm{~Hz}, I_{\text {injected }}=0.15,0.11,0.02 \mathrm{nA}$ ). (e) PRCs with individual parameters affected by cholinergic modulation modified. Parameter modifications for the respective curves are indicated in the figure $\left(I_{\text {injected }}=0.15 \mathrm{nA}, \mathrm{Hz}\right.$, firing frequencies $\left.6.7,8.1,23.6,6.6,7.0 \mathrm{~Hz}\right)$ 


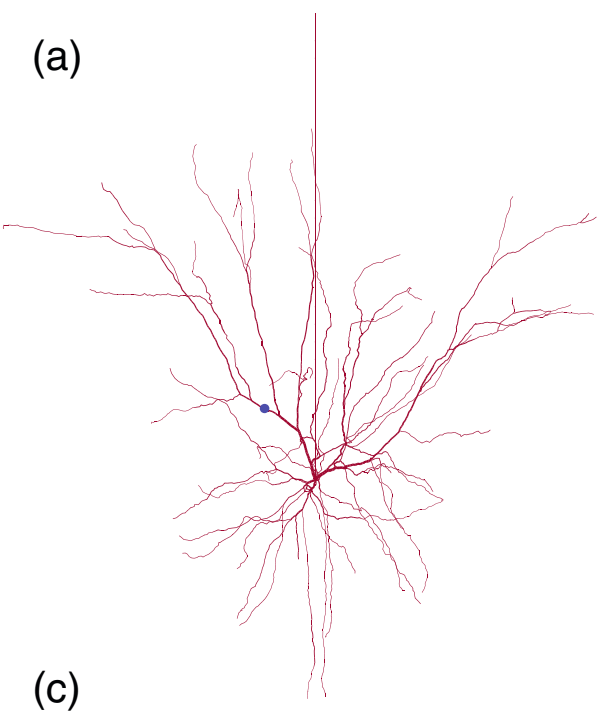

(b)

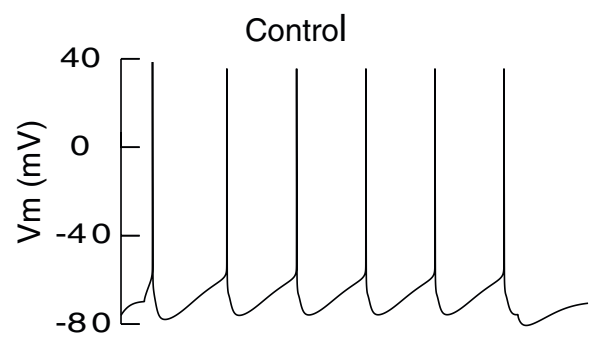

(d)
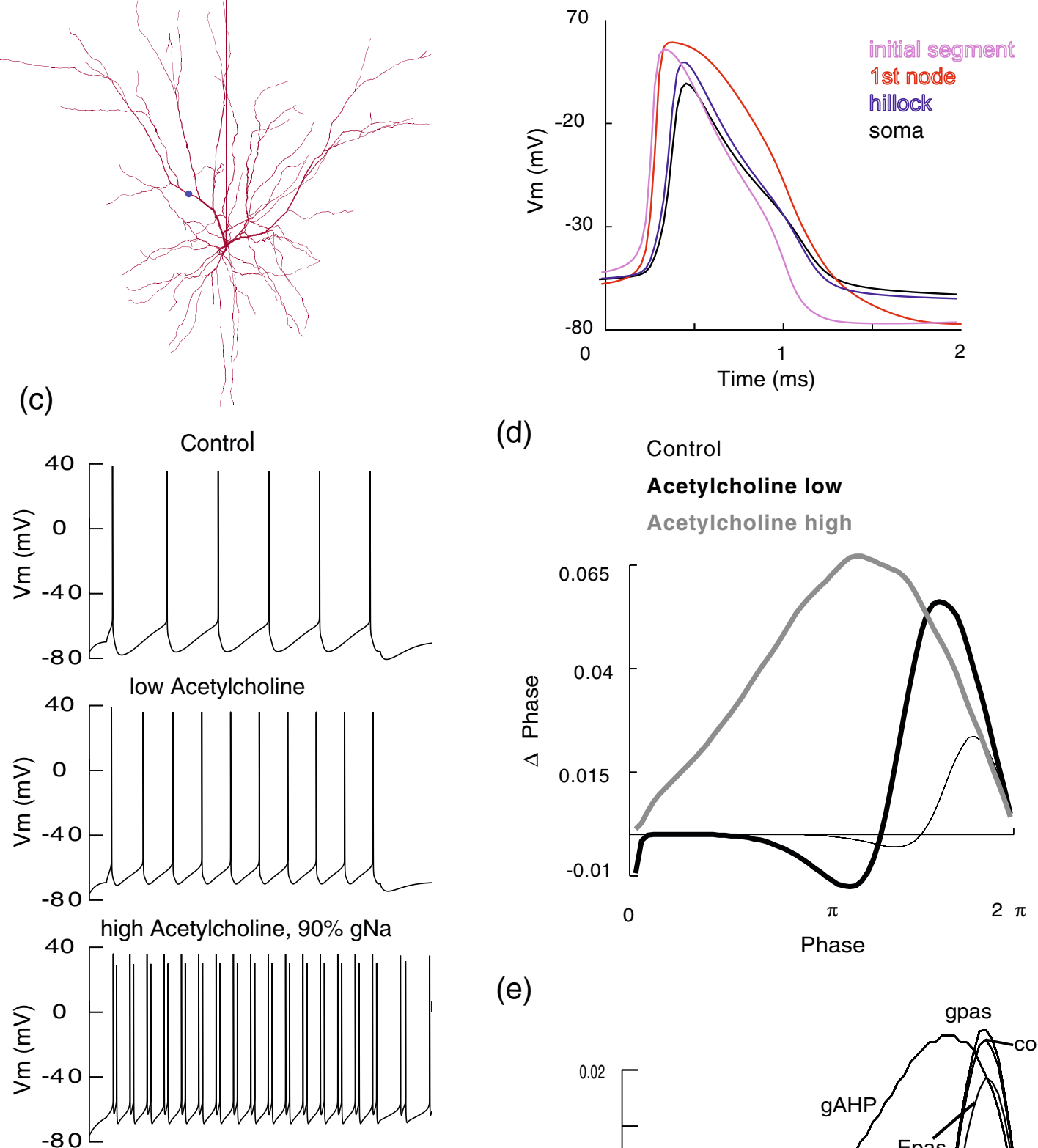

(e)

Phase

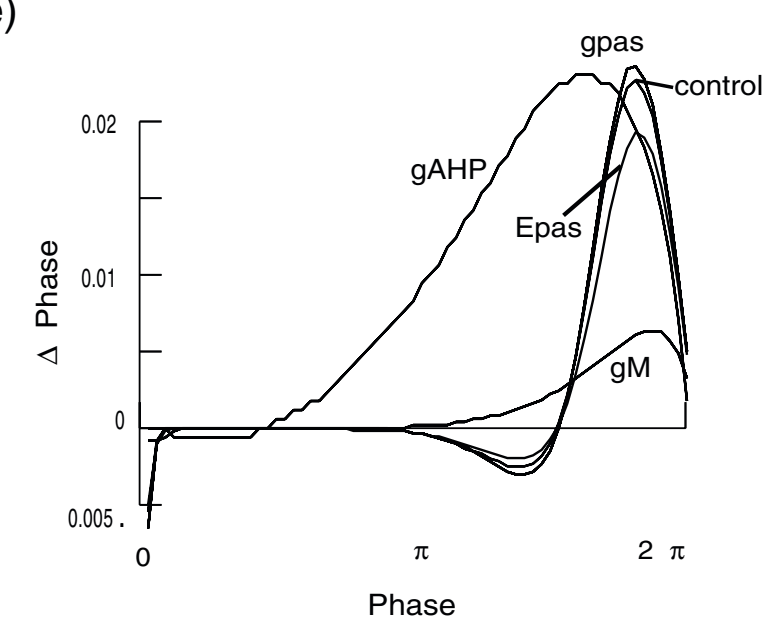


The amplitudes of the PRCs decreased with the distance of the synapse from the soma; this could be accounted for by the reduced amplitude of the EPSPs at the site of the spike initiation caused by progressively stronger dendritic cable filtering.

We next asked how a spatially non-uniform parameter change simulating cholinergic neuromodulation could change the influence of a synaptic input on the phase of neuronal spiking. The ion channel proteins carrying $I_{\mathrm{M}}$ (KCNQ) are known to be distributed relatively uniformly over the dendritic surface in related types of neurons (hippocampal CA1 neurons in culture, (Shah et al. 2002). The muscarinic acetylcholine receptors mediating the decrease in $I_{\mathrm{M}}$ conductance are also found on the dendritic
Fig. 5 Spatial effects in the complex multi-compartment neuron. (a) Location of synapses and PRCs under control conditions (thin line), low concentration cholinergic (thick line) and high concentration cholinergic parameters (gray line). (b) PRCs with control (thin line), low concentration cholinergic (thick line) and high concentration cholinergic parameters (gray line) for synapses at three different locations. Firing frequencies were kept at $7 \pm 1 \mathrm{~Hz}, I_{\text {injected }}=0.15$, 0.11, $0.02 \mathrm{nA})$. (c): Non-uniform changes of parameters simulating cholinergic effects. PRCs were determined for two synapses at the positions indicated by circles. The parameters of the dendritic conductances were changed in the black/red parts of the dendrite to simulate control conditions/ low acetylcholine (top, $I_{\text {injected }}=$ $0.03 \mathrm{nA}$, firing frequency $7.1 \mathrm{~Hz}$ ) and low/high acetylcholine (bottom, $I_{\text {injected }}=0.15 \mathrm{nA}$, firing frequency $10.4 \mathrm{~Hz}$ ) (a)

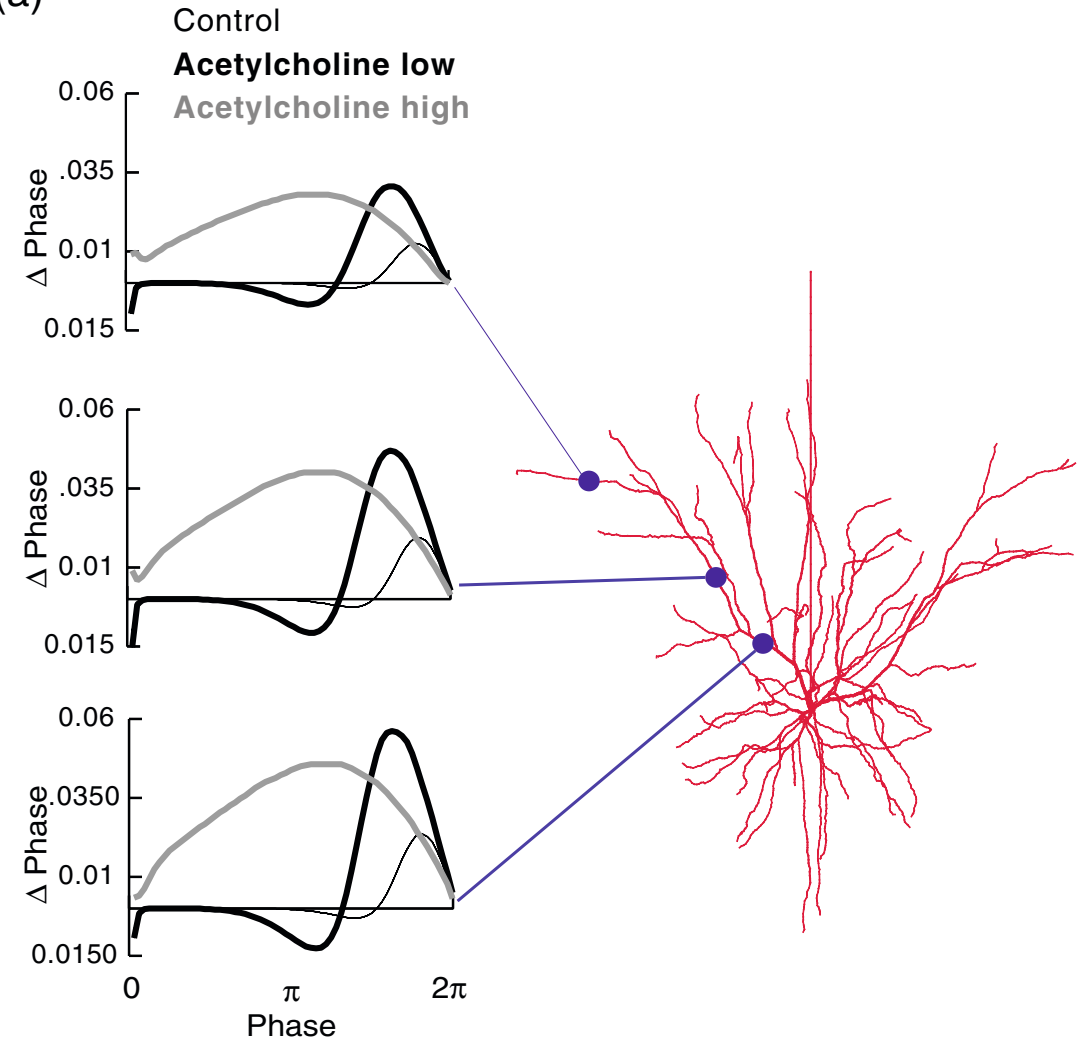

(b)

Control

Acetylcholine low

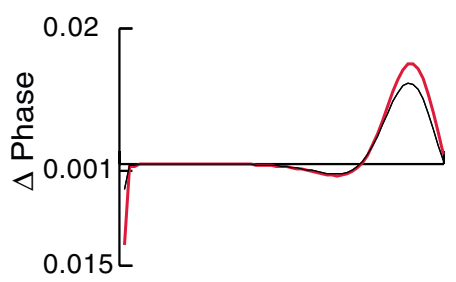

\section{Acetylcholine low} Acetylcholine high

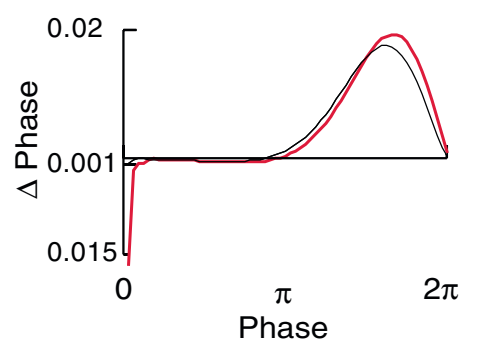

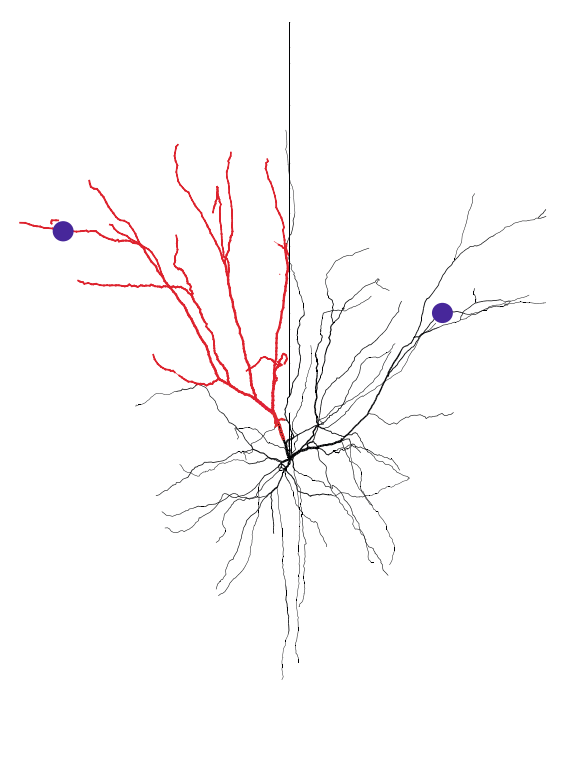


surface, and the G-protein mediated signal transduction between muscarinic receptors. On the other hand, KCNQ is highly local (spread of the signal $\sim 1 \mu \mathrm{m}$ ). Because the cholinergic projections arising from the nucleus basalis or from subtypes of cholinergic interneurons could activate muscarinic receptors in a spatially limited dendritic region, and subcellular signaling cascades mediating between them are local, cholinergic effects are likely to be localized. Similar reasoning holds for the modulation of the ion channels responsible for the leak current (GIRK).

We therefore simulated spatially heterogeneous cholinergic modulation of dendritic conductances (Fig. 5(c)). We first simulated a low concentration cholinergic modulation in a single dendritic tree ( $18 \%$ of the neuron's surface area) with no cholinergic effects in the rest of the neuron. Under these conditions, the PRC for a synapse located within and outside of the region experiencing changes was intermediate between the PRCs determined for homogeneous parameter changes. The PRC were biphasic, with the crossover point shifted to earlier phases as compared to a neuron under homogeneous control conditions. This was expected, as the dendritic tree with cholinergic parameters contained a significant fraction of the neuron's membrane surface. Surprisingly, the PRCs determined for the synapse outside the region with the cholinergically modified parameters were quite similar, although the PRC of the synapse inside the dendritic region modulated by acetylcholine had a slightly higher positive peak.

Next, we modified the parameters in the same dendritic tree to simulate a high, and in the remaining neuron a low concentration of cholinergic modulation. Again, we determined the PRCs of a synapse within and outside of this dendritic tree. As before, the PRCs were intermediate between the PRCs obtained with each parameter regime homogenously implemented over the complete dendritic tree. The negative part of the PRCs was strongly reduced. As in the previous case, the PRCs were very similar for the synapses inside and outside of the dendritic tree with different parameter modifications. The positive peak of the former PRC was slightly higher in amplitude and shifted to later phases.

The chief conclusion is that the modulation of PRC is global to a neuron. Changes of membrane properties in small dendritic regions were insufficient to switch the type of PRC of a synapse, whereas changes in large regions cause enough difference in the total currents to affect synapses outside of the region of change.

\section{Discussion}

Acetylcholine is a powerful neuromodulator that alters behavior and affects the nervous systems at both the circuit and cellular levels. In particular, some of the more prominent effects of acetylcholine at the cellar level are to reduce the spike frequency adaptation by blocking currents responsible for it, as well as to increase the resting potential of the cell, in part by a partial block of leak currents. Such modulation of cell excitability has been hypothesized to lead to changes in the structure of collective network activity in the cortex (Ermentrout et al. 2001; Crook et al. 1998b). In particular, theoretical studies have suggested that blocking adaptation can change synchronization of neurons in various frequency bands (e.g. gamma and beta; Ermentrout et al. 2001). This is supported by EEG studies in attentive vs. non-attending conditions (Fan et al. 2006), and in vitro acetylcholine modulation produces coherent oscillations in local cortical circuits (Buhl et al. 1998; Fisahn et al. 1998). In this report we focused on examining the outcome of cholinergic modulation of the various potassium and leak currents on the spike generating dynamics in model cortical pyramidal neurons by using the PRC as an assay.

We found that in a number of models the simulation of the effects of cholinergic neuromodulation on cortical pyramidal neurons led to a shift from a biphasic (type II) to a purely positive (type I) PRC. The dynamics were already present in the simplest model (theta neuron). No qualitatively new features with respect to the phenomenon of spike time resetting occur in the more sophisticated models that included all the elements necessary for spike initiation and after-hyperpolarization.

In the more complex models, cholinergic modulation revealed an unexpected interplay between the $I_{\mathrm{KAHP}}$ and $I_{\mathrm{KM}}$. Although previous studies have suggested that both currents might have similar effects on the PRC shape, producing a rightward skew in the peak (Ermentrout et al. 2001), we found that partial block of $I_{\mathrm{KAHP}}$ with $I_{\mathrm{KM}}$ fully active uncovered the negative portion of the PRC. In the low acetylcholine condition we found that PRC looked even more type II then in control; the negative lobe of the PRC increased significantly in the high acetylcholine condition, with both potassium currents the PRC switched to type I. This occurred because $I_{\mathrm{KAHP}}$ flattens out the PRC in the initial portion of the firing cycle by reducing the cells excitability in a voltage independent manner, since its activation is largely uncoupled form the voltage at the relatively hyperpolarized and partially depolarized potentials. The decrease in the excitability and cell responses to the input could occur because of the additional conductance load on the membrane by the activated $I_{\mathrm{KAHP}}$, as noted by Ermentrout et al. (2001). Thus, $I_{\mathrm{KAHP}}$ reduced the amplitude of the PRC in the initial portion of the inter-spike interval, including the negative lobe of the potentially type II PRC. Once this voltage-independent "break" on the excitability is removed, by partial block of $I_{\mathrm{KAHP}}$, the negative lobe (due to $I_{\mathrm{KM}}$ recruitment by the input induced voltage transients) was uncovered. These results suggest 
that acetylcholine should have a biphasic effect on the spike generating dynamics in pyramidal neurons, perhaps similar to the inverted U-curve suggested previously for actions of dopamine (Seamans and Yang 2004).

PRCs of spatially extended neuronal models have been determined previously (Keck et al. 2003; Goldberg et al. 2007). Here we used complex compartmental models to study what would be the effect of confining the cholinergic modulation to a particular portion of the dendritic tree. We found effect of spatially confined cholinergic changes in membrane properties were not restricted to the part of the dendritic tree subject to the changes. In fact the PRC changed from type I to type II for the full dendritic tree, even when only one of the dendrites received the simulated cholinergic modulations. This is in contrast to other electrophysiological features, such as the normalization of EPSP shapes by $I_{\mathrm{h}}$, which have been shown to be highly localized (Magee 1999).

Using the complex compartmental model we also addressed the question of how synaptic location at different dendritic site may influence the type II to type I change in the PRC. Recently, Goldberg et al. (2007) showed that active dendritic conductances can potentially shift the shape of the PRC measured at the soma, when the perturbation is delivered distally in the synapse. Their modeling studies predicted a shift from type I to type II (as the stimulation moved from the soma to the dendrite) that was confirmed by in vitro experiments. Our goal was to examine the shift from type II to type I induced by cholinergic modulation following dendritic stimulation. We found that the cholinergic modulation of the PRC type was independent of the synapse location.

Although the qualitative switch from biphasic (type II) to monophasic (type I) PRCs was seen in all models, the shape and development of the shape as a function of parameter change differed in the models used. In particular, the extrema and the zero-crossing shifted to later phases only in the theta and simple single-compartment model, not in the complex single-compartment model (the parameter dependence was not investigated in the complex multicompartment model). The integral of the PRCs under control conditions (type II) was strongly positive in the theta and simple single-compartment model. In contrast, in the complex single-compartment model and in the complex multi-compartment model, the regions under the negative and the positive parts of the PRC were quantitatively more balanced. The added features of the more complex models could also influence other phenomena, such as the integration of multiple synaptic inputs. Other extensions, such as the introduction of a stochastic component, might lead to the emergence of qualitatively new phenomena. These questions remain as topics for future investigation.

We conclude that the type and shape of the PRCs is qualitatively insensitive to changes in a number of param- eters but highly sensitive to changes in the amount of two adapting currents, $I_{\mathrm{M}}$ and $I_{\mathrm{AHP}}$. This allows a key feature of spiking, the PRC, to be manipulated without influencing other aspects of it's functioning, such as the integration of synaptic potentials. This supports our hypothesis that $I_{\mathrm{M}}$ and $I_{\mathrm{AHP}}$ cholinergic modulation is necessary and sufficient to change the PRC shape and hence the spike-generating dynamics of cortical pyramidal neurons.

An important question is what these results mean for the synchronization of neurons in the cortex in vivo. This has to be addressed in the context of the complete cortical network, which in addition to the pyramids, contains a number of types of interneurons. Different types of synchronous oscillations (delta, theta, gamma) result from different dynamics involving different currents and cell types (Tiesinga et al. 2001). The gamma oscillations, which are evoked by elevated acetylcholine (Rodriguez et al. 2004) are driven by synchronized interneural networks (Buhl et al. 1998). These interneurons then entrain the pyramids. Without interneural help, a network of reciprocally excitatory connected layer II pyramidal neurons would not synchronize when the acetylcholine concentration is high. This is predicted by theoretical results which show that neurons with a type I PRC (like layer II pyramids under the influence of acetylcholine) coupled with excitatory synapses don't synchronize (Ermentrout et al. 2001).

The picture which thus emerges is that networks of cortical layer II pyramidal neurons synchronize well when subjected to no acetylcholine and even better when exposed to low concentrations of this neuromodulator. This type of synchronization breaks down when the cholinergic neuromodulation becomes strong. Under such conditions, the pyramidal neurons are entrained into a gamma-rhythm by inhibitory interneurons. They then show no intrinsic tendency to form synchronously oscillating assemblies.

This picture is consistent with the patchy and local nature of gamma oscillations, into which pyramidal neurons are forced by local interneural circuits, and the more global nature of low-frequency oscillations (delta, theta), which result from the properties of the pyramidal neurons and their networks themselves (Tiesinga et al. 2001).

Acknowledgements This work was supported by the Deutsche Forschungsgemeinschaft (K.M.S.), the Gatsby Foundation, CNRS and Marie Curie EXT "BIND" (B.S.G.) and the Howard Hughes Medical Institute (T.J.S.). We would like to thank the organizers of and all our colleagues at the workshop "spiking neurons" at the 2004 Computational Neuroscience Meeting in Baltimore, MD, USA, where preliminary results of this work were presented.

Open Access This article is distributed under the terms of the Creative Commons Attribution Noncommercial License which permits any noncommercial use, distribution, and reproduction in any medium, provided the original author(s) and source are credited. 


\section{References}

Artola, A., \& Singer, W. (1993). Long-term depression of excitatory synaptic transmission and its relationship to long-term potentiation. Trends in Neurosciences, 16(11), 480-487. doi:10.1016/ 0166-2236(93)90081-V.

Auerbach, J. M., \& Segal, M. (1996). Muscarinic receptors mediating depression and long-term potentiation in rat hippocampus. The Journal of Physiology, 492(Pt 2), 479-493.

Buhl, E. H., Tamas, G., \& Fisahn, A. (1998). Cholinergic activation and tonic excitation induce persistent gamma oscillations in mouse somatosensory cortex in vitro. The Journal of Physiology, 513, 117-126. doi:10.1111/j.14697793.1998.117by.x.

Cantrell, A. R., Ma, J. Y., Scheuer, T., \& Catterall, W. A. (1996). Muscarinic modulation of sodium current by activation of protein kinase C in rat hippocampal neurons. Neuron, 16, 1019-1026. doi:10.1016/S0896-6273(00)80125-7.

Crook, S. M., Ermentrout, G. B., \& Bower, J. M. (1998a). Dendritic and synaptic effects in systems of coupled cortical oscillators. Journal of Computational Neuroscience, 5(3), 315-329. doi:10.1023/A:1008839112707.

Crook, S. M., Ermentrout, G. B., \& Bower, J. M. (1998b). Spike frequency adaptation affects the synchronization properties of networks of cortical oscillations. Neural Computation, 10(4), 837-854. doi:10.1162/089976698300017511.

Ermentrout, G. B. (1996). Type I membranes, phase resetting curves, and synchrony. Neural Computation, 8(5), 979-1001. doi:10.1162/neco.1996.8.5.979.

Ermentrout, G. B., Pascal, M., \& Gutkin, B. S. (2001). The effects of spike frequency adaptation and negative feedback on the synchronization of neural oscillators. Neural Computation, 13, 1285-1310. doi:10.1162/08997660152002861.

Fan, J., Byrne, J., Worden, M. S., Guise, K. G., McCandliss, B. D., Fossella, J., et al. (2006). The relation of brain oscillations to attentional networks. The Journal of Neuroscience, 27(23), 6197-6206. doi:10.1523/JNEUROSCI.1833-07.2007.

Fisahn, A., Pike, F. G., Buhl, E. H., \& Paulsen, O. (1998). Cholinergic induction of network oscillations at $40 \mathrm{~Hz}$ in the hippocampus in vitro. Nature, 394, 186-189. doi:10.1038/28179.

Goldberg, J. A., Deister, C. A., \& Wilson, C. J. (2007). Response properties and synchronization of rhythmically firing dendritic neurons. Journal of Neurophysiology, 97, 208-219. doi:10.1152/ jn.00810.2006.

Golomb, D., \& Amitai, Y. (1997). Propagating neuronal discharges in neocortical slices: computational and experimental study. Journal of Neurophysiology, 78, 1199-1211.

Gutkin, B. S., \& Ermentrout, G. B. (1998). Dynamics of membrane excitability determine interspike interval variability: a link between spike generation mechanisms and cortical spike train statistics. Neural Computation, 10(5), 1047-1065. doi:10.1162/ 089976698300017331.

Gutkin, B. S., Ermentrout, G. B., \& Reyes, A. (2005). Phase response curves determine the responses of neurons to transient inputs. Journal of Neurophysiology, 94(2), 1623-1635. doi:10.1152/ jn.00359.2004.

Hansel, D., \& Mato, G. (1995). Synchrony in excitatory neural networks. Neural Computation, 7, 307-337. doi:10.1162/ neco.1995.7.2.307

Hasselmo, M. E. (1999). Neuromodulation: acetylcholine and memory consolidation. Trends in Cognitive Sciences, 3, 351-359. doi:10.1016/S1364-6613(99)01365-0.

Hasselmo, M. E. (2006). The role of acetylcholine in learning and memory. Current Opinion in Neurobiology, 16(6), 710-715. doi:10.1016/j.conb.2006.09.002.
Hines, M. L., \& Carnevale, N. T. (1997). The NEURON simulation environment. Neural Computation, 9, 1179-1209. doi:10.1162/ neco.1997.9.6.1179.

Hines, M. L., \& Carnevale, N. T. (2000). Expanding NEURON's repertoire of mechanisms with NMODL. Neural Computation, 12, 995-1007. doi:10.1162/089976600300015475.

Hodgkin, A. L., \& Huxley, A. F. (1952). A quantitative description of membrane current and its application to conduction and excitation in nerve. The Journal of Physiology, 117, 500-544.

Izhikevich, E. M. (2007). Dynamical systems in neuroscience: the geometry of excitability and bursting. Cambridge: MIT Press.

Johnston, D., \& Wu, S. (1994). Foundations of cellular neurophysiology. Cambridge, MA: The MIT Press.

Keck, T., Netoff, T. I., \& White, J. A. (2003) Comparing dynamic responses of neurons to dendritic and somatic inputs. Society for Neuroscience Abstracts, 31.

Madison, D. V., Lancaster, B., \& Nicoll, R. A. (1987). Voltage clamp analysis of cholinergic action in the hippocampus. The Journal of Neuroscience, 7(3), 733-741.

Magee, J. C. (1999). Dendritic Ih normalizes temporal summation in hippocampal CA1 neurons. Nature Neuroscience, 2(9), 848. doi: $10.1038 / 12229$

Mainen, Z. F., \& Sejnowski, T. J. (1995). Reliability of spike timing in neocortical neurons. Science, 268, 1503-1506. doi:10.1126/ science. 7770778 .

McCormick, D. A. (1993). Actions of acetylcholine in the cerebral cortex and thalamus and implications for function. Progress in Brain Research, 98, 303-308.

Moehlis, J., Shea-Brown, E., \& Rabitz, H. (2006). Optimal inputs for phase models of spiking neurons. Journal of Computational and Nonlinear Dynamics, 1(4), 358-367. Transactions of the ASME. doi:10.1115/1.2338654.

Reyes, A. D., \& Fetz, E. E. (1993). Effects of transient depolarizing potentials on the firing rate of cat neocortical neurons. Journal of Neurophysiology, 69, 1673-1683.

Rodriguez, R., Kallenbach, U., Singer, W., \& Munk, M. H. (2004). Short- and long-term effects of cholinergic modulation on gamma oscillations and response synchronization in the visual cortex. The Journal of Neuroscience, 24(46), 10369-10378. doi:10.1523/ JNEUROSCI.1839-04.2004.

Schobesberger, H., Wheeler, D. W., \& Horn, J. P. (2000). A model for pleiotropic muscarinic potentiation of fast synaptic transmission. Journal of Neurophysiology, 83(4), 1912-1923.

Seamans, J. K., \& Yang, C. R. (2004). The principal features and mechanisms of dopamine modulation in the prefrontal cortex. Progress in Neurobiology, 74, 1-57. doi:10.1016/j. pneurobio.2004.05.006.

Shah, M. M., Mistry, M., Marsh, S. J., Brown, D. A., \& Delmas, P. (2002). Molecular correlates of the M-current in cultured rat hippocampal neurons. The Journal of Physiology, 544, 29-37. doi:10.1113/jphysiol.2002.028571.

Steriade, M. (2004). Acetylcholine systems and rhythmic activities during the waking-sleep cycle. Progress in Brain Research, 145, 179-196.

Stiefel, K., Gutkin, B. S., \& Sejnowski, T. J. (2003) Cholinergic modulation of spike generating dynamics in cortical pyramidal neurons. Society for Neuroscience Abstract, 29.

Stiefel, K. M., Tennigkeit, F., \& Singer, W. (2005). Synaptic plasticity in the absence of backpropagating spikes of layer II inputs to layer V pyramidal cells in rat visual cortex. The European Journal of Neuroscience, 21(9), 2605-2610. doi:10.1111/j.14609568.2005.04094.x.

Tiesinga, P. H. E., Fellous, J. M., Jose, J. V., \& Sejnowski, T. J. (2001). Computational model of carbachol-induced delta, theta, and gamma oscillations in the hippocampus. Hippocampus, 11, 251-274. doi:10.1002/hipo.1041. 